\title{
On Some Non-existence Results in a Semilinear Theory of the Dipolar Thermoelastic Bodies
}

\author{
Marin Marin ${ }^{1} \cdot$ Vicenţiu D. Rădulescu ${ }^{2,3}$ (D)
}

Published online: 8 July 2020

(c) The Author(s) 2020

\begin{abstract}
We consider a thermoelastic theory in which the equations that govern the evolution are linear with respect to the thermal displacement and nonlinear with regards to gradients of displacements and temperature. Our results refer to the non-existence of solutions for some mixed problems, considered in this context. We also address the instability of solutions of the considered problems. We will treat separately the case where the mechanical effects are neglected, taking into account only the thermal effect. In this case our problem has a nonlinear structure.
\end{abstract}

Keywords Thermoelasticity without energy dissipation - Dipolar bodies - Semilinear theory $\cdot$ Nonexistence $\cdot$ Instability

Mathematics Subject Classification 74A15 · 35Q74 · 74F05 · 35Q79

\section{Introduction}

It can be noticed that in the last years great attention has been paid to the phenomenon of heat transmission in elastic solids. Many studies, including those of Green and Naghdi [1-3], have proposed abandoning Fourier's classical law and considering some theories that allow the propagation of thermal waves with finite speed. In this way, the foundations of a new theory have been laid, namely thermoelasticity without energy

$凶$ Vicențiu D. Rădulescu

radulescu@inf.ucv.ro

Marin Marin

m.marin@unitbv.ro

1 Department of Mathematics and Computer Science, Transilvania University of Braşov, 500093 Braşov, Romania

2 Faculty of Applied Mathematics, AGH University of Science and Technology, al. Mickiewicza 30, 30-059 Kraków, Poland

3 Department of Mathematics, University of Craiova, Street A.I. Cuza 13, 200585 Craiova, Romania 
dissipation. After the start given by the studies of Green and Naghdi, this theory became very consistent by the presence of a large number of papers addressing all aspects of this theory of thermoelasticity without energy dissipation. There are studies that address the existence or non-existence of solutions, the uniqueness, the continuous dependence, stability or instability of solutions. In this respect, we must note the works [4-7]. All these works are dedicated to the linear case only. Our study is an attempt to address the semilinear framework, that is, models driven by equations that govern the theory of elastic dipolar bodies. These models arise in the context of thermoelasticity without energy dissipation and they are linear with respect to the thermal displacement and nonlinear with regards to temperature and gradients of displacements. But it is important to specify that in obtaining our results for the semilinear case we used some suggestions given in nonlinear studies, but in the case of isothermal elasticity. Thus, we have used methods and techniques found in the following papers: [8-13].

In our study we take into consideration an extra aspect, namely that of the dipolar structure, which is part of the modern theories dedicated to the microstructure. We have to say that the first works dedicated to the microstructure are due to Eringen (see [14, 15]). In recent years the number of papers on this subject has increased significantly. All aim to eliminate some shortcomings of classical theory. In this context, unlike the classic case, the equation of energy contains some elastic terms and it is not a parabolic type equation. Another important leap: in all theories dedicated to the microstructure, the heat waves will propagate at a finite speed. As an important component of the microstructure, the dipolar structure has attracted the attention of a large number of researchers and as a consequence, many studies have been published on this topic. In this regard, we must mention, in particular, the works [16-19]. In the studies [20-28] we find some results dedicated to different aspects of the microstructure. The methods and procedures used in the present paper have some similarities to those of classical elasticity. However, the classical results are getting worse in the new context, since the equations governing the behavior of these structures become more complicated.

In our paper we put down the equations that govern the behavior of a dipolar bodies in the context of thermoelasticity without energy dissipation. The mixed problem is completed by some specific initial and boundary conditions.

We highlight that the basic equations are linear with regards to the displacement of the heat and nonlinear with regards to temperature and gradients of displacements. Then we prove a conservation law, which will be useful in obtaining of the main results, in the first place, in obtaining an energy inequality. The result of instability of the solution is obtained for homogeneous boundary conditions. In the last part of the study we neglect the mechanical effects and get a nonlinear problem regarding the conduction of heat. We prove that some solutions cannot exist on a finite time interval.

\section{Preliminaries}

This paper is dedicated to a body with dipolar structure in the theory of thermoelasticity without energy dissipation. Our material is anisotropic and is located in a regular region $D$ from usual space $R^{3}$ and its boundary $\partial D$ is supposed be a sufficient regular surface 
to permit the application of the divergence theorem. For the closure $\bar{D}$ of the domain $D$ and we have $\bar{D}=D \cup \partial D$.

A system of Cartesian axes $O x_{i},(i=1,2,3)$ for the representation of the points is used. The tensor notation is adopted (letters in boldface). A superposed dot is used to designate the derivative with respect to time while for the differentiation regarding the coordinates $x_{i}$ we will use the notation ",i". The Einstein rule the summation over repeated indices is assumed.

Throughout this paper, the following variables are used to characterize the evolution of our dipolar material:

$$
u_{i}(t, x), \varphi_{i j}(t, x), \theta(t, x), \beta(t, x),(t, x) \in\left[0, t_{0}\right) \times D .
$$

Here $u=\left(u_{i}\right)$ is the vector of displacement and $\varphi=\left(\varphi_{i j}\right)$ is the tensor of dipolar displacement.

Also, $\theta$ is the temperature, referred to the reference configuration, and $\beta$ is the displacement of the temperature, defined by

$$
\beta(t, x)=\int_{0}^{t} \theta(\tau, x) d \tau .
$$

With the help of the variables defined in Eq. (1), we define the tensors of the strain, which have the components $\varepsilon_{i j}, \kappa_{i j}$ and $\chi_{i j k}$, by means of the geometric relations:

$$
2 \varepsilon_{i j}=u_{j, i}+u_{i, j}, \kappa_{i j}=u_{j, i}-\varphi_{i j}, \chi_{i j k}=\varphi_{i j, k} .
$$

All our considerations are made within a linear theory. Therefore, it is natural to think that Helmholtz's free energy is a quadratic form with respect to all its constitutive variables. The Helmholtz's free energy in the reference configuration will be denoted by $\Psi$. So, in accordance with the principle of energy conservation of energy, we develop in series the function $\Psi$ and we only keep the terms only until the second order. Because the reference state was assumed be free of loadings, we deduce that the Helmholtz's free energy per mass can be considered of the following form

$$
\begin{aligned}
\Psi= & \frac{1}{2} A_{i j m n} \varepsilon_{i j} \varepsilon_{m n}+D_{i j m n} \varepsilon_{i j} \kappa_{m n}+F_{i j m n r} \varepsilon_{i j} \chi_{m n r}+\frac{1}{2} B_{i j m n} \kappa_{i j} \kappa_{m n} \\
& +G_{i j m n r} \kappa_{i j} \chi_{m n r}+\frac{1}{2} C_{i j k m n r} \chi_{i j k} \chi_{m n r}-a_{i j} \varepsilon_{i j} \theta-b_{i j} \kappa_{i j} \theta-c_{i j k} \chi_{i j k} \theta-\frac{1}{2} c \theta^{2} .
\end{aligned}
$$

We will use this form of free energy used in the entropy production inequality, where we will deduce the motion equations. Also, from the same inequality the constitutive equations are obtained. These equations express the tensors of stress with the help of the tensors of deformation. We will denote the components of the stress measures by $\tau_{i j}, \sigma_{i j}$ and $\mu_{i j k}$. In this way, the constitutive equations establish a connection between the tensors $\tau_{i j}, \sigma_{i j}, \mu_{i j k}$ and the tensors $\varepsilon_{i j}, \kappa_{i j}, \chi_{i j k}$. 
We will use a procedure similar to that used by Green and Rivlin in [16], so that taking into account (4) we obtain

$$
\begin{aligned}
\tau_{i j} & =\frac{\partial \Psi}{\partial \varepsilon_{i j}}=A_{i j m n} \varepsilon_{m n}+D_{m n i j} \kappa_{m n}+F_{m n r i j} \chi_{m n r}-a_{i j} \theta, \\
\sigma_{i j} & =\frac{\partial \Psi}{\partial \kappa_{i j}}=D_{i j m n} \varepsilon_{m n}+B_{i j m n} \kappa_{m n}+G_{i j m n r} \chi_{m n r}-b_{i j} \theta, \\
\mu_{i j k} & =\frac{\partial \Psi}{\partial \chi_{i j k}}=F_{i j k m n} \varepsilon_{m n}+G_{m n i j k} \kappa_{m n}+C_{i j k m n r} \chi_{m n r}-c_{i j k} \theta, \\
\eta & =-\frac{\partial \Psi}{\partial \theta}=a_{i j} \varepsilon_{i j}+b_{i j} \kappa_{i j}+c_{i j k} \chi_{i j k}+c \theta .
\end{aligned}
$$

Here we denoted by $\eta$ the entropy per unit mass.

Also, we can deduce the main equations that govern the thermoelasticity of bodies with a dipolar structure without energy dissipation (see also [19]), namely:

- the motion equations:

$$
\begin{aligned}
\left(\tau_{i j}+\eta_{i j}\right)_{, j}+\varrho f_{i} & =\varrho \ddot{u}_{i}, \\
\mu_{i j k, i}+\eta_{j k}+\varrho g_{j k} & =I_{k r} \ddot{\varphi}_{j r}
\end{aligned}
$$

- the equation of energy is given by (see [18]):

$$
\varrho T_{0} \dot{\eta}=q_{i, i}+\varrho r
$$

The signification of the notions that we introduced in previous equations is as follows: $\varrho$-the density of mass, which is a constant; $I_{i j}$-the tensor of microinertia; $k$-the intrinsic inertia; $\varepsilon_{i j}, \kappa_{i j}, \quad \chi_{i j k}$-the strain tensors; $\tau_{i j}, \eta_{i j}, \mu_{i j k}$-the stress tensors; $h_{i}$-the components of the vector for equilibrated stress; $f_{i}$-the body forces; $g_{j k}$-the dipolar charges; $A_{i j m n}, B_{i j m n}, \ldots, a_{i j}$-the functions what describe the properties of the material in terms of elasticity. Suppose the following symmetry relations take place:

$$
\begin{aligned}
A_{i j m n} & =A_{j i m n}=A_{m n i j}, B_{i j m n}=B_{m n i j}, a_{i j}=a_{j i}, \\
C_{i j k m n r} & =C_{m n r i j k}, F_{i j k m n}=F_{i j k n m}, D_{i j m n}=D_{i j n m} .
\end{aligned}
$$

Assuming that there are no supply terms and taking into account the constitutive Eq. (5) and the kinematic Eq. (3), the Eqs. (6) and (7) receive the form

$$
\begin{aligned}
\varrho \ddot{u}_{i}= & {\left[\left(C_{i j m n}+G_{i j m n}\right) u_{n, m}+\left(G_{m n i j}+B_{i j m n}\right)\left(u_{n, m}-\varphi_{m n}\right)+\right.} \\
& \left.+\left(F_{m n r i j}+D_{i j m n r}\right) \varphi_{n r, m}-\left(a_{i j}+b_{i j}\right) \theta\right]_{, j}, \\
I_{k r} \ddot{\varphi}_{j r}= & {\left[F_{i j k m n} u_{n, m}+D_{m n i j k}\left(u_{n, m}-\varphi_{m n}\right)+A_{i j k m n r} \varphi_{n r, m}-c_{i j k} \theta\right]_{, i}+} \\
& +G_{j k m n} u_{m, n}+B_{j k m n}\left(u_{n, m}-\varphi_{m n}\right)+D_{j k m n r} \varphi_{n r, m}-b_{j k} \theta, \\
k_{i j}(\beta, j)_{, i}= & -T_{0}\left[a_{i j} \dot{u}_{i, j}+b_{i j}\left(\dot{u}_{j, i}-\dot{\varphi}_{i j}\right)+c_{i j k} \dot{\varphi}_{i j, k}+c \dot{\theta}\right] .
\end{aligned}
$$


We will complete the mixed problem for dipolar bodies in the context of thermoelasticity without energy dissipation by adding some prescribed initial conditions

$$
\begin{aligned}
u_{i}(0, x) & =u_{i}^{0}(x), \quad \dot{u}_{i}(0, x)=u_{i}^{1}(x), \\
\varphi_{j k}(0, x) & =\varphi_{j k}^{0}(x), \quad \dot{\varphi}_{j k}(0, x)=\varphi_{j k}^{1}(x), \\
\theta(0, x) & =\theta^{0}(x), \quad \beta(0, x)=\beta^{0}(x), x \in \bar{D},
\end{aligned}
$$

and, also, the given boundary conditions

$$
u_{i}=0, \varphi_{j k}=0, \theta=0 \text { on } \partial D
$$

Clearly, taking into account the definition (2) and the fact that, according to (11), $\theta=0$ on $\partial D$, we deduce that $\beta=0$ on $\partial D$.

We will denote by $\mathcal{P}$ the mixed initial boundary value problem of the theory of thermoelasticity without energy dissipation for a dipolar material over the set $\Omega_{0}=$ $D \times\left[0, t_{0}\right)$ which consists of the system of Eq. (9), for all $(t, x) \in \Omega_{0}$, the boundary conditions (11) and the initial conditions (10).

Naturally, an ordered array $\left(u_{i}, \varphi_{i j}, \theta, \beta\right)$ can be a solution for the problem $\mathcal{P}$ if it satisfies the system (9) and meet the conditions (10) and (11) of the problem.

Our first result relates to an energy conservation law corresponding to the solutions of the problem $\mathcal{P}$.

For the energy corresponding to a solution to the problem $\mathcal{P}$ we will use the notation

$$
\begin{aligned}
\mathcal{E}(t)= & \frac{1}{2} \int_{D}\left[\varrho \dot{u}_{i} \dot{u}_{i}+I_{j k} \dot{\varphi}_{j l} \dot{\varphi}_{k l}\right] d V \\
& +\frac{1}{2} \int_{D}\left[A_{i j m n} \varepsilon_{i j} \varepsilon_{m n}+2 D_{i j m n} \varepsilon_{i j} \kappa_{m n}+2 F_{i j m n r} \varepsilon_{i j} \chi_{m n r}\right. \\
& +B_{i j m n} \kappa_{i j} \kappa_{m n}+2 G_{i j m n r} \kappa_{i j} \chi_{m n r}+C_{i j k m n r} \chi_{i j k} \chi_{m n r} \\
& \left.-2 a_{i j} \varepsilon_{i j} \theta-2 b_{i j} \kappa_{i j} \theta-2 c_{i j k} \chi_{i j k} \theta-c \theta^{2}\right] d V .
\end{aligned}
$$

If we take into account (4), energy $\mathcal{E}$ receives the simplified form

$$
\mathcal{E}(t)=\frac{1}{2} \int_{D}\left[\varrho \dot{u}_{i} \dot{u}_{i}+I_{j k} \dot{\varphi}_{j l} \dot{\varphi}_{k l}\right] d V+\int_{D} \Psi d V .
$$

Proposition 1 If the ordered array $\left(u_{i}, \varphi_{i j}, \theta, \beta\right)$ is a solution of the problem $\mathcal{P}$, then the energy $\mathcal{E}$ satisfies the following conservation law:

$$
\mathcal{E}(t)=\mathcal{E}(0)
$$

Proof We multiply the Eq. (9) $)_{1}$ by $\dot{u}_{i},(9)_{2}$ by $\dot{\varphi}_{j k}$ and $(9)_{3}$ by $\dot{u}_{i}$. We sum up the obtained equalities and the equality that result is integrated on the interval $[0, t]$. If we take into account the boundary conditions from (11) and integrate on the domain $D$, we obtain the desired relation (14). 
In what follows, we will consider that the material is linear with respect to the thermal displacement gradient, that is $\beta_{i}$, and can be nonlinear with respect to the variables $u_{i, j}, \varphi_{i j, k}, \theta$. In other words, we can write the free energy in

$$
\Psi=\omega\left(u_{i, j}, \varphi_{i j, k}, \theta\right)+\frac{1}{2} K_{i j} \beta_{, i} \beta_{, j},
$$

where the tensor $K_{i j}$ is positively defined.

As a consequence, the law of energy conservation from (14) becomes

$$
\begin{aligned}
\mathcal{E}(t)= & \frac{1}{2} \int_{D}\left[\varrho \dot{u}_{i} \dot{u}_{i}+I_{j k} \dot{\varphi}_{j l} \dot{\varphi}_{k l}+K_{i j} \beta_{, i} \beta_{, i}\right] d V \\
& +\int_{D}\left[\omega\left(u_{i, j}, \varphi_{i j, k}, \theta\right)+\eta \theta\right] d V=\mathcal{E}(0) .
\end{aligned}
$$

Based on the considerations made in the papers $[10,12]$ in the case of classical elasticity, without taking into account the thermal effect, we can replace the Eq. (16) with the following inequality,

$$
\mathcal{E}(t) \leq \mathcal{E}(0)
$$

Also, if energy $\mathcal{E}$ is associated not with a classical solution of the system (9), but with a weak solution, it is more appropriate to use the inequality (17) than the Eq. (16).

We now aim to achieve a result of instability. For this, we will first consider the following boundary values problem

$$
\begin{aligned}
\left(K_{i j} q_{, j}\right)_{, i} & =-\eta\left(u_{i, j}^{0}, \varphi_{i j, k}^{0}, \theta^{0}\right), \\
q & =0, \quad x \in \partial D
\end{aligned}
$$

where the initial data $\left(u_{i, j}^{0}, \varphi_{i j, k}^{0}, \theta^{0}\right)$ are introduced in (10).

If $q$ is a solution to the problem (18), then we introduce the notation

$$
\vartheta=\int_{0}^{t} \beta(\tau) d \tau+q .
$$

In the following we will consider that energy has the following form:

$$
\mathcal{E}_{1}(t)=\frac{1}{2} \int_{D}\left[\varrho u_{i} u_{i}+I_{j k} \varphi_{j l} \varphi_{k l}+K_{i j} \vartheta_{, i} \vartheta_{, i}\right] d V .
$$

Theorem 1 We consider that a weak solution of the problem $\mathcal{P}$ is the ordered array $\left(u_{i}, \varphi_{i j}, \theta, \beta\right)$. If, in addition, the following condition is met

$$
\int_{D}\left(-\frac{\partial \omega}{\partial u_{i, j}} u_{i, j}-\frac{\partial \omega}{\partial \varphi_{i j, k}} \varphi_{i j, k}+\frac{\partial \omega}{\partial \theta} \theta\right) d V \geq 0,
$$


then the energy $\mathcal{E}_{1}(t)$ becomes unbounded, that is, the equilibrium state becomes unstable.

Proof By direct calculations, from (19) we are led to the inequality

$$
\begin{aligned}
\frac{d^{2} \mathcal{E}_{1}}{d t^{2}}= & \int_{D}\left[\varrho u_{i} u_{i}+I_{j k} \varphi_{j l} \varphi_{k l}+K_{i j} \beta_{, i} \beta, j\right] d V \\
& +\int_{D}\left(-\frac{\partial \omega}{\partial u_{i, j}} u_{i, j}-\frac{\partial \omega}{\partial \varphi_{i j, k}} \varphi_{i j, k}+\frac{\partial \omega}{\partial \theta} \theta\right) d V .
\end{aligned}
$$

We take into account that $\varrho>0$ and the tensors $I_{i j}$ and $K_{i j}$ are positively defined. Considering the condition (20) and using Schwarz's inequality, we obtain the inequality

$$
\frac{d^{2} \mathcal{E}_{1}^{1 / 2}}{d t^{2}} \geq 0
$$

which involves the condition

$$
\mathcal{E}_{1}^{1 / 2}(t) \geq \frac{t \frac{d \mathcal{E}_{1}}{d t}(0)+2 \mathcal{E}_{1}(0)}{\mathcal{E}_{1}^{1 / 2}(0)}
$$

It is clear that if $\frac{d \mathcal{E}_{1}}{d t}(0)>0$, then, the condition (23) guarantees that energy $\mathcal{E}_{1}^{1 / 2}(t)$ becomes unbounded, which ends the demonstration of the theorem.

Now we will address a non-existence result of the solution of the problem $\mathcal{P}$. To this aim we will construct an auxiliary function $F(t)(t)$, with the help of the function $\mathcal{E}_{1}($ from (19)), by

$$
F(t)=\mathcal{E}_{1}(t)+\frac{1}{2} \alpha\left(t+t_{0}\right)^{2}, \alpha \geq 0
$$

Theorem 2 Suppose that the inequality (22) is satisfied and for $\zeta>2$ the following condition is checked

$$
\int_{D}\left[-\frac{\partial \omega}{\partial u_{i, j}} u_{i, j}-\frac{\partial \omega}{\partial \varphi_{i j, k}} \varphi_{i j, k}+\frac{\partial \omega}{\partial \theta} \theta+\zeta(\omega+\eta \theta)\right] d V \geq 0 .
$$

Then, there is no a solution $\left(u_{i}, \varphi_{i j}, \theta, \beta\right)$ of the problem $\mathcal{P}$ which should be defined in finite time.

Proof By direct calculations we obtain

$$
\frac{d F(t)}{d t}=\int_{D}\left(\varrho u_{i} \dot{u}_{i}+I_{j k} \varphi_{j l} \dot{\varphi}_{k l}+K_{i j} \vartheta_{, i} \beta_{, j}\right) d V+\alpha\left(t+t_{0}\right) .
$$


By using the energy inequality, from (26) we are led to

$$
\begin{aligned}
\frac{d^{2} F(t)}{d t^{2}} \geq & \frac{\zeta+2}{2} \int_{D}\left(\varrho \dot{u}_{i} \dot{u}_{i}+I_{j k} \dot{\varphi}_{j l} \dot{\varphi}_{k l}+K_{i j} \beta_{, i} \beta, j\right) d V \\
& +\int_{D}\left(-\frac{\partial \omega}{\partial u_{i, j}} u_{i, j}-\frac{\partial \omega}{\partial \varphi_{i j, k}} \varphi_{i j, k}+\frac{\partial \omega}{\partial \theta} \theta+\zeta(\omega+\eta \theta)\right) d V+(\alpha-\zeta \mathcal{E}(0)),
\end{aligned}
$$

and from here, with the help of Schwarz's inequality, we get

$$
\frac{d^{2} F(t)}{d t^{2}} F(t)-\frac{\zeta+2}{4}\left(\frac{d F(t)}{d t}\right)^{2} \geq-\zeta(\alpha+\mathcal{E}(0)) F(t) .
$$

Taking into account that $\zeta>2$, we can reverse in inequality (28), so that using the notation $p=(\zeta-2) / 2$, inequality can be reformulated so

$$
\frac{d^{2} F^{-p}(t)}{d t^{2}} F(t) \leq 2 p(2 p+1) F^{-(p+)}(t)(\alpha+\mathcal{E}(0)) \text {. }
$$

If we give to $\alpha$ a particular value, namely $\alpha=-\mathcal{E}(0)$, then from inequality (28) we obtain

$$
\frac{d^{2} F^{-p}(t)}{d t^{2}} \leq 0
$$

which ensures the concavity of the function $F^{-p}(t)$.

Taking into account the definition (25) we deduce that

$$
F(t) \geq F(0)\left(1-p t \frac{d F}{d t}(0) F(0)^{-1}\right)^{-1 / p}, t \in\left[0, t_{0}\right) .
$$

We choose a $t_{0}$ large enough to guarantee the positivity of the derivative $d F / d t$. As a consequence, the lower bound from the right-hand side of the inequality (30) allows to the function $F$ to grow as much as possible in a finite time. Thus, the proof of the theorem is completed.

In the last part of our study, we will neglect the mechanical effects. In other words, we consider that the function $W$ which designates the free energy is only depending on temperature $\theta$ and of the gradient of the displacement of the heat $\beta_{, i}$, that is,

$$
W=\omega(\theta)+\frac{1}{2} K_{i j} \beta_{, i} \beta_{, j} .
$$

As such, we approach the problem (nonlinear) of the conduction of heat, consisting of the nonlinear equation

$$
\frac{d}{d t}\left(\frac{\partial W}{\partial \theta}\right)+\left(\frac{\partial W}{\partial \beta_{, i}}\right)=0
$$


the following initial conditions

$$
\theta(0, x)=\theta^{0}(x), \beta(0, x)=\beta^{0}(x), x \in \bar{D},
$$

and the homogeneous boundary condition

$$
\beta=0 \text { on } \partial D \text {. }
$$

In order to determine the conditions in which the mixed problem (32)-(34) admits a solution, it is recommended the works [29,30].

We will show that, under specific conditions, the problem (32)-(34) can not admit a solution in finite time.

To make evaluations on the solutions to the problem (32)-(34) we introduce the function $G(t)$, defined with the help of the free energy $W(t)$ from (31), through

$$
G(t)=\int_{D} \frac{\partial W}{\partial \theta} \beta d V
$$

Let us suppose that the free energy $W(t)$ satisfies the following condition

$$
W+\frac{\partial W}{\partial \beta_{, i}} \beta_{, i} \geq 0
$$

Theorem 3 We consider a solution of problem (32)-(34) for which the initial data (33) is chosen so that the energy $\mathcal{E}$ is negative.

If the function $G(t)$ attached to this solution satisfies the condition (36), then the solution ceases to exist for $t \rightarrow \infty$.

Proof In fact, we have to show that the solution becomes unbounded if $t \rightarrow \infty$.

If we take into account the condition (34) and use the theorem of divergence, from (32) we are led to the relation:

$$
G(t)=\int_{0}^{t} \int_{D}\left(\frac{\partial W}{\partial \theta} \theta+\frac{\partial W}{\partial \beta_{, i}} \beta_{, i}\right) d V d \tau+G(0) .
$$

Clearly, by direct derivation in (37) we obtain the following identity

$$
\frac{d G(t)}{d t}=\int_{D}\left(\frac{\partial W}{\partial \theta} \theta+\frac{\partial W}{\partial \beta_{, i}} \beta_{, i}\right) d V,
$$

and from here, with the help of energy inequality, we are led to the next estimate

$$
\frac{d G(t)}{d t} \geq \int_{D}\left(W+\frac{\partial W}{\partial \beta_{, i}} \beta_{, i}\right) d V-\mathcal{E}(0) .
$$

Finally, taking into account the hypothesis (36), from (38) we get the inequality

$$
G(t) \geq-G(0)-t \mathcal{E}(0),
$$


which ends the proof of the theorem.

\section{Conclusions}

In the analysis developed in the present paper, we approach a semilinear initial boundary value problem describing thermoelastic dipolar bodies without energy dissipation. In our setting, the basic equations that describe the problem are linear with regard to the thermal displacement and nonlinear with respect to the gradients of displacements and temperature. This approach is original and it cannot be found at present for environments with dipolar structure. Our results refer to the non-existence of solutions for some mixed problems, considered in this context. Another feature of this paper is that we also address the instability of the solutions of the considered problems. We have paid special attention to the case when the mechanical effects are absent. It is noteworthy that if the mechanical effects are neglected, then the theory becomes a nonlinear one. However, we have managed to achieve both a result regarding the non-existence of the solutions and a result concerning instability issues.

Acknowledgements The authors thank both anonymous reviewers for the careful reading of the manuscript and for proposing pertinent remarks that have improved the initial version of this paper.

Open Access This article is licensed under a Creative Commons Attribution 4.0 International License, which permits use, sharing, adaptation, distribution and reproduction in any medium or format, as long as you give appropriate credit to the original author(s) and the source, provide a link to the Creative Commons licence, and indicate if changes were made. The images or other third party material in this article are included in the article's Creative Commons licence, unless indicated otherwise in a credit line to the material. If material is not included in the article's Creative Commons licence and your intended use is not permitted by statutory regulation or exceeds the permitted use, you will need to obtain permission directly from the copyright holder. To view a copy of this licence, visit http://creativecommons.org/licenses/by/4.0/.

\section{References}

1. Green, A.E., Naghdi, P.M.: On undamped heat waves in an elastic solid. J. Therm. Stress. 15, 253-264 (1992)

2. Green, A.E., Naghdi, P.M.: Thermoelasticity without energy dissipation. J. Elast. 31, 189-208 (1993)

3. Green, A.E., Naghdi, P.M.: A re-examination of the basic postulates of themomechanics. Proc. R. Soc. Lond. A 432, 171-194 (1991)

4. Iesan, D.: On the theory of thermoelasticity without energy dissipation. J. Therm. Stress. 21, 295-307 (1998)

5. Nappa, L.: Spatial decay estimates for the evolution equations of linear thermoelasticity without energy dissipation. J. Therm. Stress. 21, 581-592 (1998)

6. Quintanilla, R., Straughan, B.: Growth and uniqueness in thermoelasticity. Proc. R. Soc. Lond. (Ser. A) 456, 1419-1429 (2000)

7. Quintanilla, R.: Existence in thermoelasticity without energy dissipation. J. Therm. Stress. 25(2), 195202 (2002)

8. Ames, K.A., Straughan, B.: Non-standard and Improperly Posed Problems. Academic Press, San Diego (1997)

9. Flavin, J.N., Knops, R.J., Payne, L.E.: Asymptotic behaviour of solutions to semilinear elliptic equations on the half-cylinder. Z. Angew. Math. Phys. (ZAMP) 43, 405-421 (1992)

10. Knops, R.J.: Instability an the ill-posed Cauchy problem. In: Hopkins, H.G., Sewell, M.J. (eds.) Elasticity in Mechanics of Solids. Pergamon Press, Oxford (1982) 
11. Knops, RJ., Straughan, B.: Non-existence of global solutions to nonlinear Cauchy problems arising in mechanics in Trends. In: Applications of Pure Mathematics to Mechanics, Pitman, London, (1976)

12. Knops, R.J., Levine, H.A., Payne, L.E.: Nonexistence, instability and growth theorems for solutions of a class of abstract nonlinear equations with applications to nonlinear elastodynamics. Arch. Ration. Mech. Anal. 55, 52-72 (1974)

13. Levine, H.A.: Instability and nonexistence of global solutions to nonlinear wave equations of the form $P u_{t t}=-A u+F(u)$. Trans. Am. Math.Soc 190, 1-21 (1974)

14. Eringen, A.C.: Theory of micromorphic materials with memory. Int. J. Eng. Sci. 10, 623-641 (1972)

15. Eringen, A.C.: Theory of thermo-microstretch elastic solids. Int. J. Eng. Sci. 28, 1291-1301 (1990)

16. Green, A.E., Rivlin, R.S.: Multipolar continuum mechanics. Arch. Ration. Mech. Anal. 17, 113-147 (1964)

17. Fried, E., Gurtin, M.E.: Thermomechanics of the interface between a body and its environment. Contin. Mech. Thermodyn. 19(5), 253-271 (2007)

18. Marin, M., Rădulescu, V.: A variational approach for the mixed problem in the elastostatics of bodies with dipolar structure. Mediterr. J. Math. 15(221), 1-12 (2018)

19. Mindlin, R.D.: Micro-structure in linear elasticity. Arch. Ration. Mech. Anal. 16, 51-78 (1964)

20. Abbas, I.A.: Eigenvalue approach for an unbounded medium with a spherical cavity based upon twotemperature generalized thermoelastic theory. J Mech Sci Technol. 28(10), 4193-4198 (2014)

21. Abbas, I.A., Marin, M.: Analytical solution of thermoelastic interaction in a half-space by pulsed laser heating. Physica E 87, 254-260 (2017)

22. Abd-Elaziz, E.M., et al.: On the effect of Thomson and initial stress in a thermo-porous elastic solid under GN electromagnetic theory. Symmetry 11(3), 413 (2019)

23. Bhatti, M.M., et al.: Numerical study of heat transfer and Hall current impact on peristaltic propulsion of particle-fluid suspension with compliant wall properties. Mod. Phys. Lett. B 33(35), 1950439 (2019)

24. Marin, M., Craciun, E.M., Pop, N.: Considerations on mixed initial-boundary value problems for micropolar porous bodies. Dyn. Syst. Appl. 25(1-2), 175-196 (2016)

25. Othman, M.I.A., Marin, M.: Effect of thermal loading due to laser pulse on thermoelas-tic porous medium under G-N theory. Results Phys. 7, 3863-3872 (2017)

26. Riaz, A., et al.: Study of heat and mass transfer in the Eyring-Powell model of fluid propagating peristaltically through a rectangular compliant channel. Heat Transf. Res. 50(16), 1539-1560 (2019)

27. Teodorescu-Draghicescu, H., et al.: Finite element method analysis of some fibre-reinforced composite laminates. Optoelectron. Adv. Mat. 5(7), 782-785 (2011)

28. Vlase, S., et al.: Behavior of multiphase fiber-reinforced polymers under short time cyclic loading. Optoelectron. Adv. Mat. 5(3-4), 419-423 (2011)

29. Fichera, G.: Linear theories of elasticity and thermoelasticity. In: Truesdell, C. (ed.) Existence Theorems in Elasticity, pp. 347-424. Springer, Berlin (1973)

30. Marin, M., Öchsner, A.: Essentials of Partial Differential Equations with Applications. Springer, Cham (2019)

Publisher's Note Springer Nature remains neutral with regard to jurisdictional claims in published maps and institutional affiliations. 